NEUES AUS DER INDUSTRIE

\section{Aktivitätenberichte von Inteco}

\section{China}

Im Frühjahr 2014 wurde die zukünftige Zusammenarbeit der INTECO Gruppe und der Henan Xibao Metallurgy Materials Group Co. Ltd. beschlossen und vertraglich festgehalten. Xibao, eine Firmengruppe mit 3500 Mitarbeitern, ist einer der größten führenden Produzenten von metallurgischen Verbrauchsgütern am chinesischen Markt.

Die Produktpalette des im chinesischen Henan angesiedelten Produktionsbetriebes reicht von Ferrolegierungen über Feuerfestmaterialien und Gießpulver bis zu verschiedenen Schlacken, insbesondere auch vorgeschmolzenen Schlacken für den ESU-Betrieb.

210 Stahlwerke in über 20 Ländern beziehen bereits die Produkte von Xibao.

Nutznießer des gemeinsamen Kooperationsvertrages sind beide Unternehmen. TECTRADE, eine der Tochterfirmen INTECOs, wird für Xibao die exklusive Vermarktung von ESUSchlacke und ausgewählten Produkten in Europa übernehmen. Als Weltmarktführer im Bau von ESU-Anlagen wird man bei INTECO die vorhandenen Kontakte nützen und sich $u$. a. auf den Vertrieb von ESU-Schlacke konzentrieren. Xibao wird die von INTECO und deren Tochterfirma in Dalian eigens für den chinesischen Markt konzipierte "Smart ESU“- Anlage verkaufstechnisch unterstützen.

Auch Dr. Harald Holzgruber, Geschäftsführer von INTECO, freut sich über die Zusammenarbeit: „Xibao und INTECO werden beide von den Netzwerken des jeweils anderen profitieren. Zudem ist ein hohes gegenseitiges Vertrauen gegeben, da die Produkte von beiden Firmen für hohe Qualität und Kundenzufriedenheit stehen."

\section{Schweden}

Im März erhielt INTECO von der schwedischen Firma Uddeholm den Auftrag über die Lieferung einer $30 \mathrm{t}$ Elektroschlackeumschmelzanlage (ESU).
Dies ist zwar die erste INTECO Anlage, aber bereits der zehnte ESU Ofen, der am Standort in Hagfors in Betrieb gehen wird. Uddeholm ist der größte Produzent von ESU umgeschmolzenem Werkzeugstahl weltweit. Mit der Standtiegelanlage des österreichischen Anlagenbauers sollen bei einem maximalen Druck von 3 bar Edelstahlblöcke mit einem maximalen Gewicht von $30 \mathrm{t}$ und einem maximalen Durchmesser von $1250 \mathrm{~mm}$ produziert werden können. Zusätzlich besitzt die Anlage alle dem Stand der Technik entsprechenden Eigenschaften, wie zum Beispiel Umschmelzen unter Schutzgas, koaxiale Hochstromführung und ein komplett innovatives Kontrollsystem der letzten Generation.

Im Rahmen der Verhandlungen wurde auch die Option des Kaufs einer zweiten identen Anlage besprochen abhängig von der Marktsituation in näherer Zukunft. Das gesamte INTECO Team freut sich auf die Zusammenarbeit und Herausforderungen im Rahmen dieses Projektes und wird diese Gelegenheit nutzen, die führende Position als Anbieter von Elektroschlackeumschmelzanlagen unter Beweis zu stellen.

\section{Mexiko}

Das mexikanische Unternehmen Frisa, einer der führenden Betriebe für Freiformschmiedestücke, erteilte INTECO den Auftrag über die Lieferung eines Spezialstahlwerkes.

Das neue Werk besteht aus einem Elektrolichtbogenofen, einem Pfannenofen, einer Vakuumentgasungsanlage, einer Legierungsanlage und einem Blockgießbetrieb. Um die hohe Qualität der Anlagen sicherzustellen, wird das Engineering von INTECO special melting technologies $\mathrm{GmbH}$, Leitbetrieb der INTECO Gruppe, erstellt. Die Fertigung erfolgt in bewährten Partnerbetrieben. Daneben werden Kernkomponenten auf höchstem technischem Niveau von Firmen aus der INTECO Gruppe geliefert:

Process Technology Int., kurz PTI, wird den Elektrolichtbogenofen mit Brennern (PTI's JETburnerTM), Einblasesystemen sowie Probenahmemanipulatoren ausstatten. Weiters kommt PTI's SwingDoorTM mit einge- bautem Brenner als Schlackentür zum Einsatz.

Sowohl der Elektrolichtbogenofen als auch der Pfannenofen werden mit dem Elektrodenregler ISEC von INTECO atec automation betrieben. ISEC zeichnet sich durch hohe Energieeffizienz sowie einfache Konfiguration und Bedienung.

Das neue Stahlwerk wird Anfang 2016 in Betrieb gehen.

\section{Russland}

Im Juni 2014 wurde INTECO mit der Planung und Lieferung einer 6 t VAR Anlage vom russischen Spezialstahlhersteller Ruspolymet beauftragt.

Hierbei handelt es sich bereits um den zweiten Folgeauftrag für INTECO: Auf die im Jahr 2012 verkaufte VIM Anlage folgte im Herbst 2013 eine $10 \mathrm{t}$ ESU Anlage und jetzt im Juni 2014 der VAR Ofen. Die ersten beiden Anlagen befinden sich bereits in der Montagephase bzw. knapp vor Auslieferung, und es gibt kein besseres Indiz für die Zufriedenheit des Kunden mit der bisherigen Projektabwicklung seitens INTECO als dieser neuer Auftrag.

Dieser hochmoderne Vakuumlichtbogenofen wird die im VIMOfen hergestellten Elektroden erneut umschmelzen und wird somit Ruspolymet die Gelegenheit geben, in Zukunft neue Märkte erschließen zu können. Die erzeugten Blöcke werden einen maximalen Durchmesser von rund $700 \mathrm{~mm}$ bei einem Gewicht von ca. $6 \mathrm{t}$ haben. Mit einem von INTECO völlig neu entwickelten Level 2 System wird Ruspolymet in der Lage sein, die gesamte Prozesskette (VIM, ESU, VAR) zu verwalten, metallurgisch zu führen und zu optimieren.

Derzeit laufen bereits weitere Gespräche über neue Projekte, und beide Firmen überlegen sich eine strategische Partnerschaft einzugehen.

INTECO special melting technologies $\mathrm{gmbH}$ Wienerstr.25

8600 Bruck a. d. Mur

03862531100

www.inteco.at 